\title{
Phenotypic Overlap of Cardiac Sodium Channelopathies Individual-Specific or Mutation-Specific?
}

\author{
Naomasa Makita, MD
}

\begin{abstract}
Mutations in the cardiac sodium channel gene SCN5A are responsible for a spectrum of hereditary arrhythmias, including type-3 long QT syndrome (LQT3), Brugada syndrome (BrS), conduction disturbance and sinus node dysfunction. These syndromes were originally regarded as independent entities with distinct clinical manifestations and biophysical properties, but recent evidence shows considerable clinical overlap, implying a new disease entity known as an overlap syndrome of cardiac sodium channelopathy. Class IC sodium-channel blockers often induced the BrS phenotype in some patients with LQT3, confirming the clinical overlap of LQT3 and BrS. It also raises a concern about the safety of the class IC drug and questions about the determinants of overlap. Here, an overview is given of current knowledge on the clinical features, prevalence, and molecular and biophysical mechanisms underlying overlap syndrome to gain more insight into this complex issue and generate better therapeutic strategies for patient management. (Circ J 2009; 73: 810-817)
\end{abstract}

Key Words: Brugada syndrome; Flecainide; Long QT syndrome; Overlap syndrome; SCN5A

\section{Structure-Function of Cardiac Sodium Channels}

The voltage-gated sodium $(\mathrm{Na})$ channel is responsible for the rapid upstroke of the action potential in most of the excitable tissues, and plays a pivotal role in the initiation, propagation, and maintenance of normal cardiac rhythm. The cardiac Na channel comprises the most prevalent poreforming $\alpha$-subunit (Nav1.5) encoded by the gene SCN5A located on chromosome 3p21 and auxiliary $\beta$ subunits (Nav $\beta 1-N a v \beta 4)$ encoded by the genes $S C N 1 B-S C N 4 B$, respectively. The $\alpha$-subunits have a 4 -fold symmetry macromolecule consisting of structurally homologous domains (D1-D4) each containing 6 membrane-spanning segments (S1-S6) and a region (S5-S6 loop) controlling ion selectivity and permeation. The positively charged S4 segment of each domain functions as a voltage sensor!,2

The $\mathrm{Na}$ channels switch between 3 functional states (closed, open, inactivated), depending on the membrane potential. Membrane depolarization causes a rapid rise in local Na permeability because of opening (activation) of the $\mathrm{Na}$ channels from their resting closed state. Normally, activation of the Na channels is transient, because of inactivation, another gating process mediated by structures located on the cytoplasmic face of the channel protein (mainly the D3-D4 linker). The Na channels cannot reopen until the membrane is repolarized and they recover from inactivation. Membrane repolarization is achieved by fast inactivation of the Na channels and is augmented by activation of

(Received January 6, 2009; revised manuscript received January 26 2009; accepted January 27, 2009; released online March 31, 2009) Department of Cardiovascular Medicine, Hokkaido University Graduate School of Medicine, Sapporo, Japan

Mailing address: Naomasa Makita, MD, Department of Cardiovascular Medicine, Hokkaido University Graduate School of Medicine, Kita15, Nishi-7, Kita-ku, Sapporo 060-8638, Japan. E-mail: makitan03@ yahoo.co.jp

All rights are reserved to the Japanese Circulation Society. For permissions, please e-mail: cj@j-circ.or.jp voltage-gated $\mathrm{K}$ channels. Activation, inactivation, and recovery from inactivation occur within a few milliseconds. In addition to these rapid gating transitions, the $\mathrm{Na}$ channels are also susceptible to slower inactivating processes (slow inactivation) if the membrane remains depolarized for a longer time. These slower events may contribute to the availability of active channels under various physiological conditions.

\section{Genetics of Cardiac Sodium Channelopathies}

Mutations of SCN5A are responsible for a spectrum of hereditary arrhythmias, including type-3 congenial long QT syndrome (LQTS; LQT3),3,4 acquired LQTS5, Brugada syndrome $(\mathrm{BrS} ; \mathrm{BrS} 1)^{4,6}$ cardiac conduction disturbance (CCD)? congenital sick sinus syndrome (SSS), atrial standstill,-11 AV block, ${ }^{12}$ sudden infant death syndrome, ${ }^{13-15}$ and familial atrial fibrillation ${ }^{16-18}$ (Table 1). In addition to these primary electrical diseases, which usually do not have structural abnormalities, SCN5A mutations have also been reported in patients with dilated cardiomyopathy!6,19 Moreover, recent genetic studies have indicated that mutations

Table 1. Inherited Cardiac Sodium Channelopathies

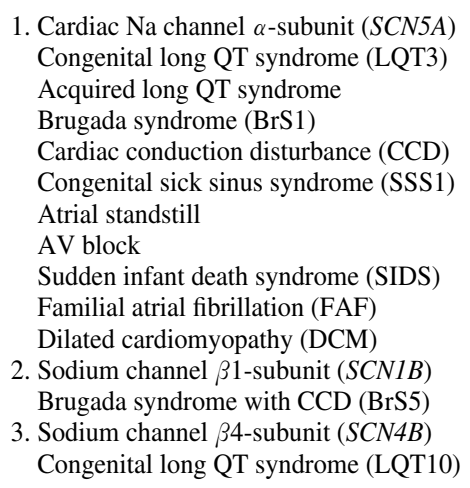


Table 2. Mutations in Overlapping Cardiac Sodium Channelopathies

\begin{tabular}{llc}
\hline Clinical manifestation & \multicolumn{1}{c}{$S C N 5 A$ and $S C N 1 B(*)$ mutations } & References \\
\hline BrS+LQT3 $( \pm$ CCD $)$ & V411M, D1114N, W1191X, I1350T, $\Delta$ K1500, & $22,35,37,42-46$ \\
& SKPQ, R1612P, P2066A & $8,32,33,35,40,41,47,48$ \\
BrS+LQT3+SSS $( \pm$ CCD $)$ & G1262S, $\Delta$ F1617, E1784K, 1795insD & $31,49,50$ \\
BrS+SSS & E161K, T187I, E1225K, K1479, K1578fs. R1623X & 11 \\
BrS+AS & R367H & $50-54$ \\
BrS+CCD & P336L, D356N, R376H, N406S, G752R, \\
& F861fs951X, E867X, G1319V & $20,30,50,51,55-57$ \\
DCM (+/-CCD+SSS+AF) & G1406R, I1660V, S1710L, S1812X, E87Q*, Y179X* & $9,16,19$ \\
CCD+SSS/AS & G1408R & 8 \\
AS+AF & L212P & 10 \\
LQT3+CCD & P1332L, V1763M, M1766L, V1777M, P2005A & $58-65$ \\
\hline
\end{tabular}

BrS, Brugada syndrome; LQT3, type-3 long QT syndrome; SSS, sick sinus syndrome; CCD, cardiac conduction disturbance; AS, atrial standstill; DCM, dilated cardiomyopathy; AF, atrial fibrillation.
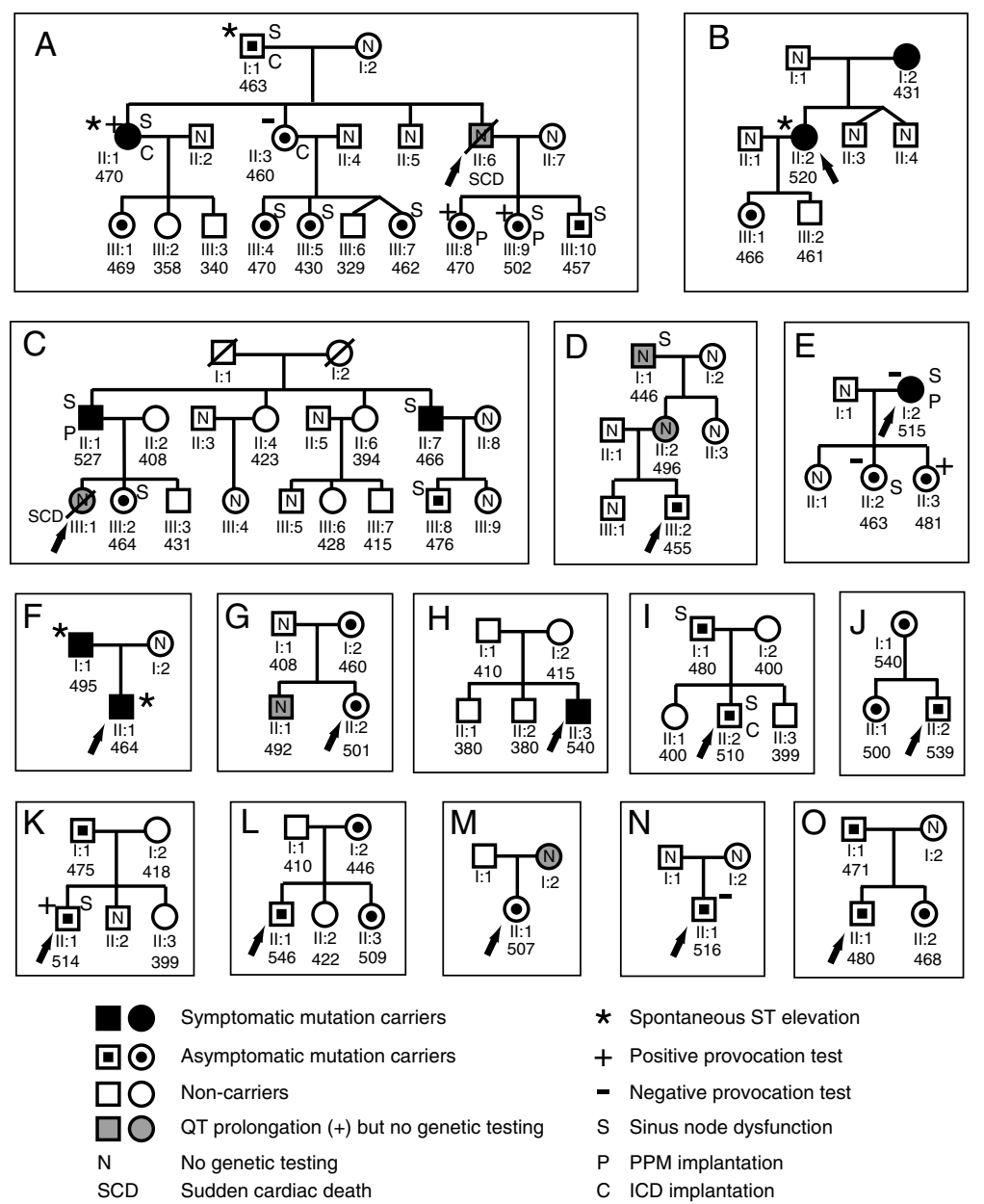

Figure 1. Pedigrees of 15 LQT3 families (A-O) carrying E1784K are shown. Probands are indicated by an arrow; 10 symptomatic mutation carriers, shown by the filled symbols, had episodes of syncope $(n=9)$ and unexplained palpitations ( $\mathrm{n}=1 ; \mathbf{B}, \mathrm{II}: 2)$. Asymptomatic mutation carriers $(\mathrm{n}=31)$ are shown as symbols with a dot, and shaded symbols are the individuals with QT prolongation who declined genetic testing or sudden cardiac death victims (SCD; A, II:6 and $\mathbf{C}$, III:1). *Individuals exhibiting ST elevation in the right precordial leads. Values for QTc intervals are given beneath each symbol. The Na-channel provocation test was positive in individuals with $+(\mathbf{A}$, II: $1 ; \mathbf{A}$, III: $: 8 ; \mathbf{A}$, III:9; $\mathbf{E}$, II:3; K, II:1), and negative in the individuals with - (A, II:3; E, I:2; E, II:2; N, II:1). Individuals with a positive and negative Na-channel blocker provocation test are shown as + and -, respectively. (Modified with permission from Makita et al. J Clin Invest 2008; 118: $2219-222941$ )

in the Na channel $\beta$-subunit genes, $S C N 1 B$ and $S C N 4 B$, are associated with type-5 $\mathrm{BrS}$ complicated with $\mathrm{CCD}(\mathrm{BrS} 5)^{20}$ and type-10 LQTS (LQT10), respectively.

\section{Biophysical Properties of Cardiac Sodium Channelopathies}

Congenital LQTS is characterized by prolongation of the QT interval on surface ECGs and an increased risk of potentially fatal ventricular arrhythmias, especially torsade de pointes 21 The QT interval is determined by the cardiac action potential, which is orchestrated by a fine balance between inward and outward currents expressed in myocardial cells. After the first identification in 1995 of the SCN5A mutation $\triangle \mathrm{KPQ}$, comprising a deletion of 3 conserved amino acids, 1505-1507, in the cytoplasmic D3-D4 linker22 more than 100 distinct $S C N 5 A$ mutations responsible for LQT3 have been reported. The common in vitro consequence of most of these mutations is a persistent Na current during the action potential plateau because of destabilized fast Na channel inactivation? This failure of fast inactivation shifts the ionic balance during the plateau phase toward the 


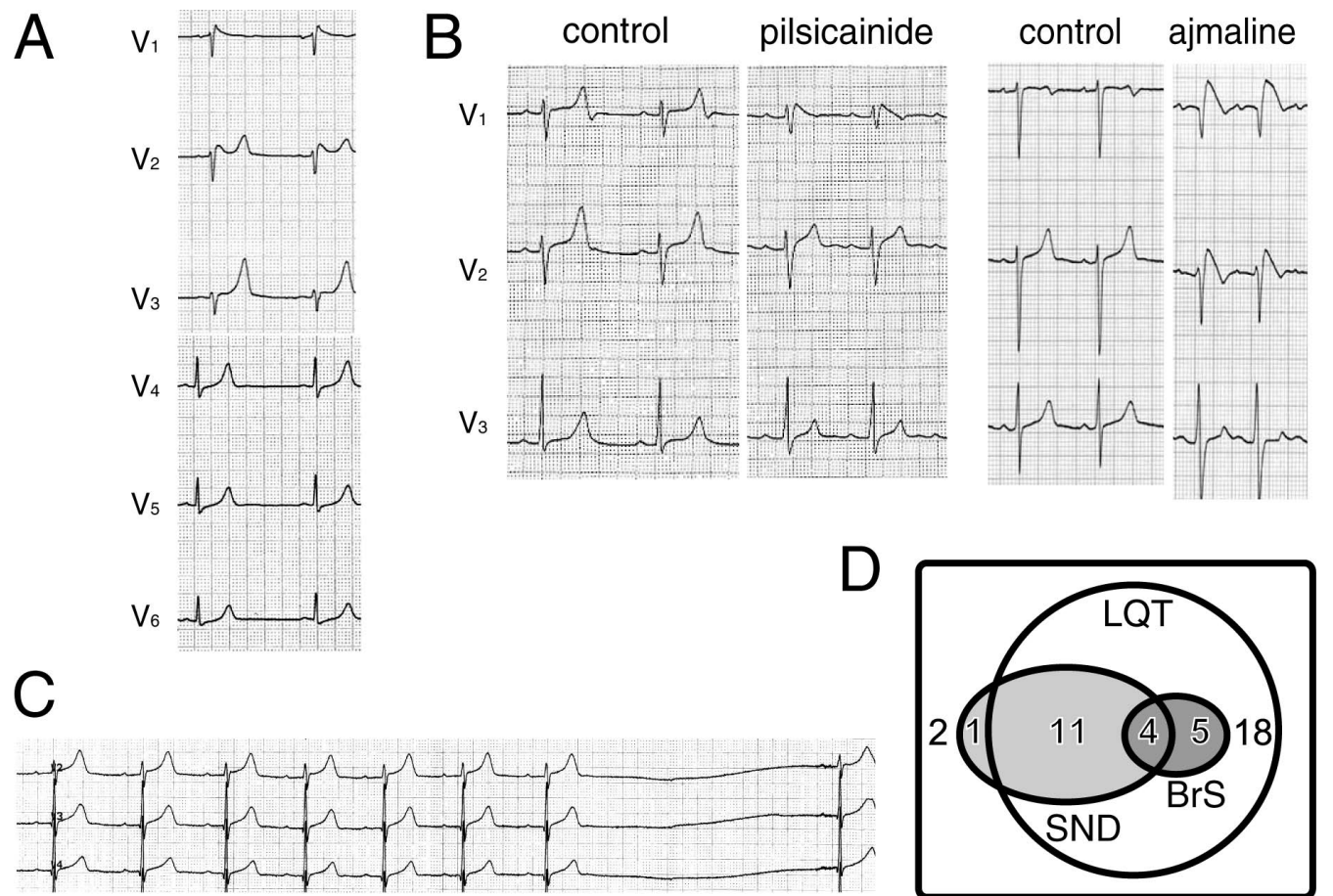

Figure 2. ECG characteristics of E1784K mutation carriers. QT prolongation $(\mathrm{QTc}=470 \mathrm{~ms})$ and spontaneous saddleback type ST elevation observed in the right precordial leads in a carrier (Figure 1A, II:1). (A) ECG recordings before and after the Na-channel blocker provocation test. Pilsicainide (Left, patient K, II:1) induced a coved-type ST elevation in V1 and the QTc was concomitantly shortened (QTc: control $495 \mathrm{~ms}$, pilsicainide $459 \mathrm{~ms}$ ). Ajmaline (Right, patient A, III:9) also induced a coved-type ST elevation in $\mathrm{V}_{1}$ and $\mathrm{V}_{2}$ and QTc shortening (control $501 \mathrm{~ms}$, ajmaline $490 \mathrm{~ms}$ ). (B) SND demonstrated by a $3.9 \mathrm{~s}$ sinus arrest in a carrier (A, I:1). (C) Venn diagram representing electrophysiological manifestation of 41 SCN5A-E1784K mutation carriers: 38 carriers had an abnormally long QTc; 3 individuals had a normal QTc, and 1 exhibited sinus node dysfunction (SND) only. SND and Brugada syndrome were observed in 15 and 9 individuals, respectively, with 4 displaying both phenotypes. (Modified with permission from Makita et al. J Clin Invest 2008; 118: 2219-222941)

inward current (gain-of-function) and delays repolarization, thus increasing the action potential duration and the corresponding QT interval. Na channel blockers, such as mexiletine (class IB) or flecainide (class IC), shorten the QT in patients with LQT3 by blocking of this persistent current, ${ }^{23-25}$ therefore are theoretically useful in the management of affected patients.

$\mathrm{BrS}$ is another primary electrical disorder without underlying structural heart diseases characterized by the covedtype ST elevation in the right precordial leads. ${ }^{26,27}$ It predisposes affected individuals to ventricular fibrillation (VF). Mutations in SCN5A are identified in $20-30 \%$ patients with $\mathrm{BrS}$, and most of the heterologously expressed mutant $\mathrm{Na}$ channels exhibit biophysical abnormalities resulting in reduced cardiac Na current (loss-of-function) ${ }^{28}$ Reduced $\mathrm{Na}$ current is thought to exaggerate differences in the action potential duration between the inner (endocardium) and outer (epicardium) layers of ventricular muscle, thereby favoring substrate promoting reentrant arrhythmias. Lossof-function of the cardiac Na channels is either owing to (1) haplo-insufficiency because of non-functional mutations, (2) impaired altered channel-gating properties, including enhanced inactivation, disruption of activation and impaired recovery from inactivation, or (3) impaired intracellular trafficking and decreased membrane surface expression of the channel molecules.

\section{Clinical Overlap of Cardiac Sodium Channelopathies}

SCN5A mutations with loss-of-function properties have also been identified in patients with $\mathrm{CCD}^{7,29} \mathrm{SSS}$, and atrial standstill, and numbers of reports have shown that the mutation carriers tend to exhibit overlapping clinical properties of these syndromes ${ }^{30,31}$ (Table 2). Importantly, such lossof-function properties are apparently opposite to those described in LQT3 (gain-of-function), and different SCN5A mutations were initially linked to separate arrhythmias syndromes. Surprisingly, some LQT3 patients display ECG findings characteristic of $\mathrm{BrS}$, suggesting that a single mutation can be associated with a wide spectrum of disease phenotypes. Such phenotypic overlap between LQT3 and BrS was first reported in a large multigenerational Dutch family with an insertion mutation $1795 \mathrm{ins}$, in which the mutation carriers showed ECG features of both LQT3 and $\mathrm{BrS}$, and sinus node dysfunction (SND)?32,33 Importantly, Na channel block in the overlap phenotype shortens QT, but exacerbated the ST segment elevation BrS phenotype, and thus enhances arrhythmia risk ${ }^{33}$ Biophysical studies demonstrated that the mutant channels displayed enhancement of both closed-state inactivation and slow inactivation, which was thought to sensitize carriers to the BrS phenotype during flecainide therapy ${ }^{34}$ in addition to the persistent Na current, a hallmark Na channel property of LQT3.

The overlap between the LQT3 and BrS phenotypes was also reported in other $S C N 5 A$ mutations such as $\triangle \mathrm{KPQ}, 35,36$ 
A

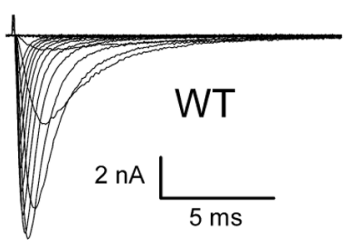

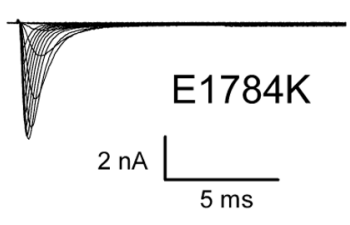

B
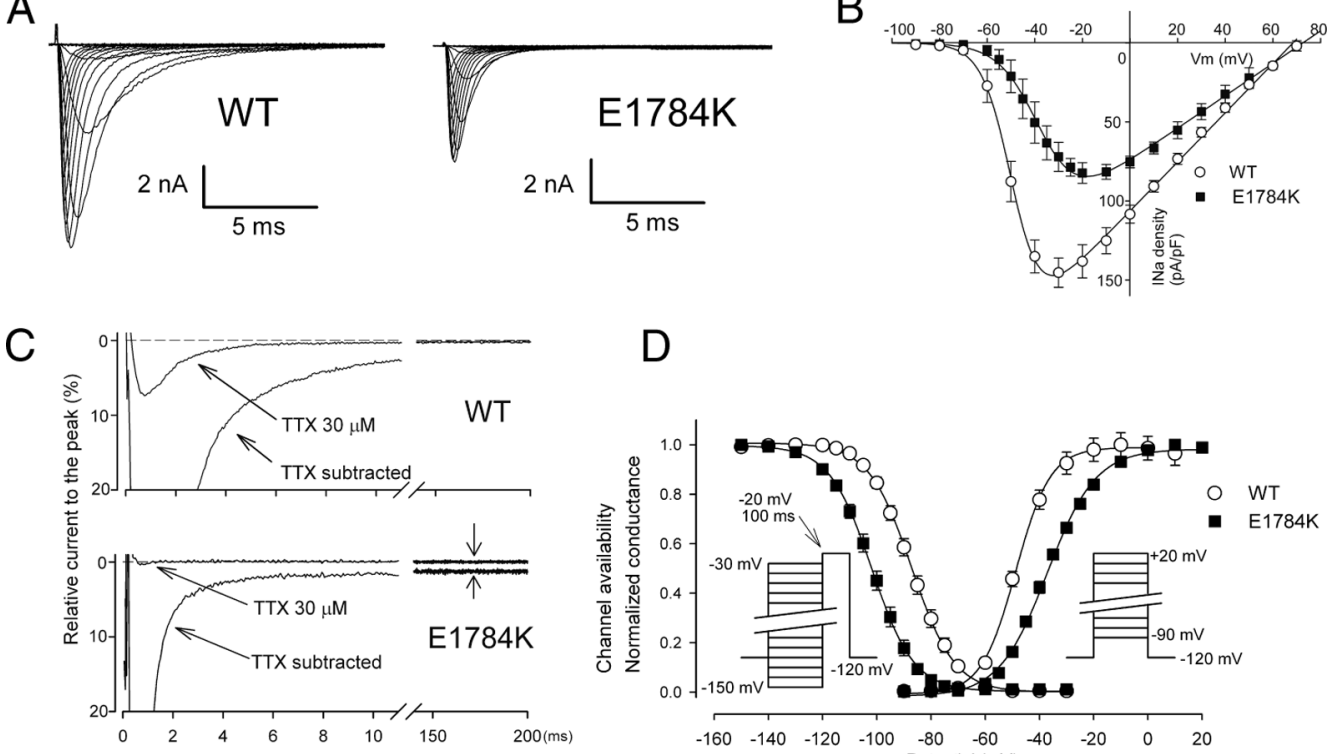

D

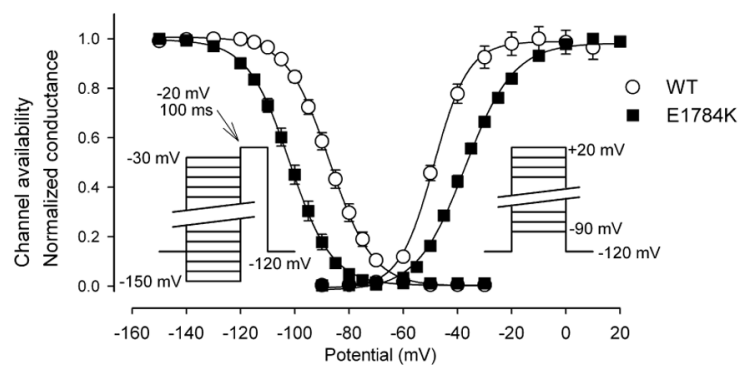

Figure 3. Properties of E1784K whole-cell current. (A) Representative whole-cell current traces obtained from tsA-201 cells transfected with either wild-type (WT) or E1784K Na channels; all studies were conducted in cells co-transfected with human sodium channel $\beta 1$-subunits. Currents were recorded from a holding potential of $-120 \mathrm{mV}$ and stepped from $-90 \mathrm{mV}$ to $+90 \mathrm{mV}$ for $20 \mathrm{~ms}$ in $10 \mathrm{mV}$ increments. (B) Current-voltage relationship. Current was normalized to cell capacitance to give a measure of Na current density. (C) Na currents were recorded with a test pulse potential of $-20 \mathrm{mV}$ from a holding potential of $-120 \mathrm{mV}$ showed prominent tetrodotoxin-sensitive late Na current (shown with arrows) and the faster decay in E1784K. (D) Steady-state availability for fast inactivation and the conductance-voltage relationship were measured with standard pulse protocols shown in the inset. Curves were fit with the Boltzmann equation. The voltagedependence of steady-state fast inactivation and activation were significantly shifted in the hyperpolarizing $(-15.0 \mathrm{mV})$ and depolarizing $(+12.5 \mathrm{mV})$ directions, respectively. (Modified with permission from Makita et al. J Clin Invest 2008; 118: $2219-222941$ )

$\mathrm{E} 1784 \mathrm{~K}^{35}$ and $\Delta \mathrm{K} 1500{ }^{37}$ Priori et al showed the additional evidence for the elusive link between these 2 clinical syndromes by the fact that the class IC sodium channel blocker flecainide induced ST-segment elevation in the right precordial leads not only in patients with BrS, but also with LQT335 Of 13 patients in 7 LQT3 families (SCN5A mutations of V411M, T1304M, $\Delta \mathrm{K} 1500, \Delta \mathrm{KPQ}, \mathrm{R} 1626 \mathrm{P}$, E1784K, P2006A), 6 showed flecainide-induced ST elevation. However, they failed to identify the determinants of flecainide-induced ST elevation in patients with LQT3. In fact, they assumed that the clinical overlap appeared to be individual-specific, rather than gene-specific or mutationspecific, most likely because the size of their patients was rather small. Nonetheless, these observations raise a concern about the safety of class IC drug therapy in LQT3 patients and questions about the underlying mechanisms.

Phenotypic variability in LQT3 has thus far been reported sporadically or only within a single kindred 32,33 Therefore, it is unclear whether development of the BrS phenotype in a patient with LQT3 is solely determined by the biophysical properties of the mutant channel, or by co-inherited genetic variations, gender, ethnicity, or other environmental factors. One approach to dissecting such phenotypic variability is to perform a clinical assessment of individuals with multiple pedigrees from genetically heterogeneous populations with the same mutation.

\section{Clinical Phenotypes in 15 LQT3 Families With SCN5A-E1784K}

From 7 institutions in Japan, Italy, Germany, UK and the
US, 44 genotyped LQT3 families with different ethnic backgrounds were enrolled (Asian 20, Caucasian 24). E1784K was the most prevalent SCN5A mutation, identified in 15 families (34\%). Two probands died suddenly, and 66 of the 93 surviving members underwent genetic testing. There were 41 mutation carriers and 25 non-carriers, and QTc was significantly prolonged in the carriers.

Spontaneous ST elevation in the right precordial leads occurred in 5/41 mutation carriers (Figure 1; coved-type: $\mathrm{n}=1$, saddle-back type: $\mathrm{n}=4$, Figure 2A). Nine mutation carriers without diagnostic ST elevation at baseline underwent provocation with flecainide, ajmaline, or pilsicainide, and the test was positive (coved-type ST elevation, Figure 2B) in 5 (shown with + in Figure 1). Thus, the diagnosis of $\mathrm{BrS}$ was established in 9/41 mutation carriers (1 individual showed spontaneous saddle-back ST elevation which was converted to coved-type by ajmaline).

SND was common in the cohort, presenting in $16 / 41$ mutation carriers (Figure 2C), and 4 of these 16 carriers with SND also exhibited the BrS phenotype (Figures 2B, D). Moreover, 1 carrier showed SND without manifesting QT prolongation or ST elevation. Four patients received a permanent pacemaker and 3 received an implantable cardioverter defibrillator.

\section{Biophysical Properties and Membrane Trafficking of E1784K}

Whole-cell patch clamp recording showed that E1784K has the following biophysical abnormalities: (1) significantly smaller peak current density, (2) persistent Na current, (3) 
A

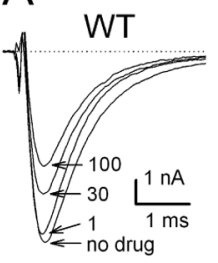

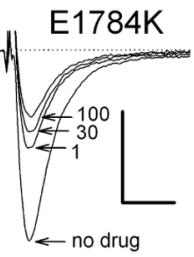
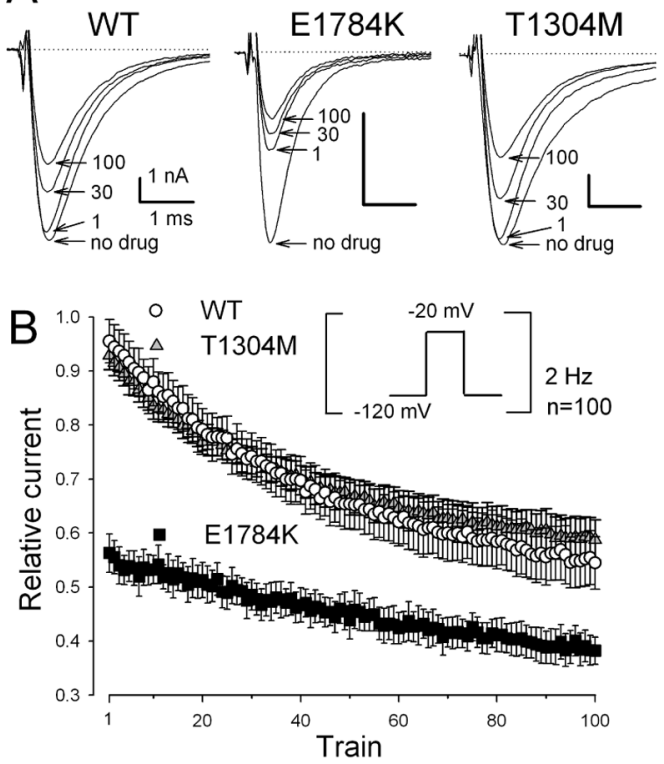

C

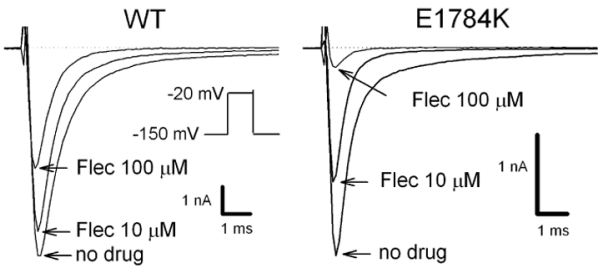

$\mathrm{D}$

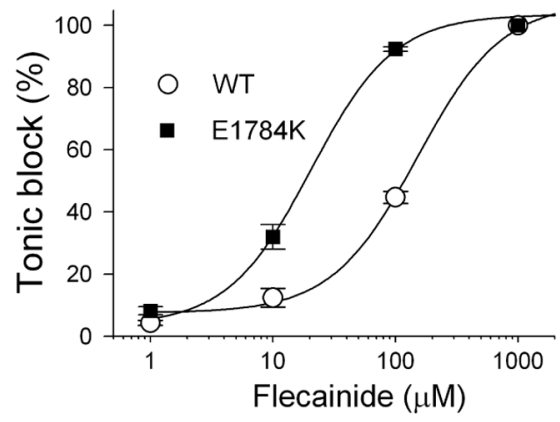

Figure 4. Tonic block, use-dependent block, and the dose-dependence of flecainide. (A) Representative current traces of wild-type (WT), E1784K, and T1304M before and after $10 \mu \mathrm{mol} / \mathrm{L}$ flecainide. A train of 100 pulses (to $-20 \mathrm{mV}$ for $20 \mathrm{~ms}$ ) was applied at $2 \mathrm{~Hz}$ from a holding potential of $-120 \mathrm{mV}$. Numbers indicate the $1^{\text {st }}(1), 30^{\text {th }}$ (30), and $100^{\text {th }}(100)$ pulse of the train. (B) Time course of the peak current levels after application of $10 \mu \mathrm{mol} / \mathrm{L}$ flecainide. Peak current levels recorded with each pulse were normalized to the baseline prior to flecainide. (C) Representative steady-state current traces of WT and $\mathrm{E} 1784 \mathrm{~K}$ before and after flecainide $(10$ and $100 \mu \mathrm{mol} / \mathrm{L})$. Cells were depolarized by $-20 \mathrm{mV}$ from a holding potential of $-150 \mathrm{mV}$. (D) Concentration-response curve for flecainide-induced tonic block in wild-type (WT) and E1784K. The normalized peak currents were fitted to the Hill equation. The IC50 values, representing dissociation constants for resting state were: WT, $150.3 \mu \mathrm{mol} / \mathrm{L} ; \mathrm{E} 1784 \mathrm{~K}, 20.4 \mu \mathrm{mol} / \mathrm{L}$. Thus, the mutant channel was far more sensitive to tonic block by flecainide. (Modified with permission from Makita et al. J Clin Invest 2008; 118: 2219-222941.)

Table 3. Reported Biophysical Properties of LQT3 Mutations

\begin{tabular}{|c|c|c|c|c|c|}
\hline & \multicolumn{5}{|c|}{ SCN5A mutations } \\
\hline & 1795 insD & $\Delta \mathrm{KPQ}$ & $\Delta \mathrm{K} 1500$ & E1784K & $\mathrm{T} 1304 \mathrm{M}$ \\
\hline \multicolumn{6}{|l|}{ Clinical features } \\
\hline QT prolongation & + & + & + & + & + \\
\hline ST elevation ${ }^{\dagger}$ & + & + & + & + & - \\
\hline Sinus node dysfunction & + & + & + & + & - \\
\hline \multicolumn{6}{|c|}{ Biophysical and pharmacological properties } \\
\hline Persistent $\mathrm{Na}$ current & + & + & + & + & + \\
\hline Shift of $\mathrm{V}_{1 / 2}$ (inactivation) & Negative & Negative & Negative & Negative & Positive \\
\hline Shift of $V_{1 / 2}$ (activation) & Positive & Positive & Positive & Positive & Positive \\
\hline Current decay & Slower & Faster & $\longleftrightarrow$ & Faster & Faster \\
\hline Recovery from inactivation & Slower & Faster & ND & $\longleftrightarrow$ & Faster \\
\hline Slow inactivation & Enhanced & $\longleftrightarrow$ & ND & Enhanced & $\longleftrightarrow$ \\
\hline Tonic block by flecainide & Enhanced & Enhanced & Enhanced & Enhanced & $\longleftrightarrow$ \\
\hline UDB by flecainide & Enhanced & Enhanced & ND & Diminished & $\longleftrightarrow$ \\
\hline References & $32,33,38,66$ & $3,41,67$ & 37 & 40,41 & 13,41 \\
\hline
\end{tabular}

${ }_{\dagger}$ Spontaneous or Na-channel blocker-induced ST elevation; $\longleftrightarrow$ comparable to wild type.

$\mathrm{ND}$, not determined.

Modified with permission from Makita et al. J Clin Invest 2008; 118: 2219-222941

significantly faster macroscopic current decay, (4) hyperpolarizing shift of the steady-state inactivation, (5) significant depolarizing shift in the voltage-dependence of activation, and (6) normal recovery from inactivation (Figure 3). Furthermore, using a $\mathrm{Na}$ channel plasmid construct with an extracellular FLAG epitope, membrane trafficking of E1784K determined by a confocal laser scanning microscopy was comparable to the wild-type (WT). These observations provide strong evidence that the loss-of-function properties displayed by E1784K are most likely attributable to the aforementioned changes in gating properties rather than a change in channel density.

\section{Molecular Mechanisms of Enhanced Flecainide Sensitivity}

Class IC drug challenge test was positive in $56 \%$ patients with E1784K. Tonic block and use-dependent block by flecainide in WT and E1784K channels have been investigated, and compared with those of T1304M, a mutation that did not 
show ST elevation during flecainide challenge test ${ }^{35}$ Cells transfected with WT, E1784K, or T1304M were depolarized by $2 \mathrm{~Hz}$ pulse trains in the absence or presence of $10 \mu \mathrm{mol} / \mathrm{L}$ flecainide. During exposure to flecainide, peak currents normalized to predrug baseline were progressively reduced by the repetitive pulses (Figures $\mathbf{4 A}, \mathbf{B}$ ). There was a remarkable difference in the extent of first pulse (tonic) block that was only $4.5 \pm 4.0 \%$ for WT, and $7.1 \pm 2.7 \%$ for $\mathrm{T} 1304 \mathrm{M}$, compared with substantial tonic block in E1784K (43.7士 $8.0 \%, \mathrm{P}<0.001)$. Conversely, use-dependent block, determined by the difference in peak current values between the $1^{\text {st }}$ and $100^{\text {th }}$ test pulses relative to the $1^{\text {st }}$ pulse, was slightly attenuated in E1784K. The net effect of flecainide after a train of 100 pulses was significantly greater in E1784K than in WT, but not in T1304M. Moreover, dose-response curves for flecainide block measured at a holding potential of $-150 \mathrm{mV}$ (thus representing drug affinity for the resting state) showed that the E1784K channels were 7.5-fold more sensitive to resting-state block by flecainide than were the WT channels $(\mathrm{IC} 50: \mathrm{WT}=150.3 \mu \mathrm{mol} / \mathrm{L}, \mathrm{E} 1784 \mathrm{~K}=20.4 \mu \mathrm{mol} / \mathrm{L}$ ) (Figures 4C,D). These results indicate that the E1784K channels are much more sensitive to blocking by flecainide than are the WT and T1304M channels, and that this augmented sensitivity is attributable to enhanced tonic block rather than a change in use-dependent block.

\section{Functional Determinants of LQT3 Associated With BrS and SND}

To explore the functional determinants for the phenotypic overlap of BrS in LQT3 patients, the biophysical and pharmacological properties of reported LQT3 mutations were compared, and features commonly and specifically observed in those manifesting a BrS phenotype were sought (Table 3). The overlapping phenotype (LQT3 and BrS) has been previously reported for $1795 \mathrm{insD}, 32,33 \Delta \mathrm{KPQ}^{35,36} \Delta \mathrm{K} 150037$ and E1784K 35 In contrast, a carrier of T1304M did not show ST elevation during a flecainide test ${ }^{35}$ Similarly, SND has been reported in carriers of the same SCN5A mutations, 1795 insD ${ }^{38} \Delta \mathrm{KPQ}^{39}{ }^{39} \Delta \mathrm{K} 1500,{ }^{37} \mathrm{E} 1784 \mathrm{~K},{ }^{40}$ and $\mathrm{D} 1790 \mathrm{G},{ }^{24}$ but not in other SCN5A mutations, including T1304M. Thus, it is plausible to speculate that the biophysical characteristics common to these mutations, but not found in T1304M, are channel properties responsible for evoking the mixed phenotypes of BrS and SND in patients with LQT3. Table 3 is a comparison of the functional properties of E1784K, and those reported for $1795 \mathrm{insD}, \Delta \mathrm{KPQ}, \Delta \mathrm{K} 1500, \mathrm{E} 1784 \mathrm{~K}$, and T1304M. ${ }^{13}$ Among the biophysical properties listed in Table 3, both the negative-shift in steady-state inactivation, and the enhanced tonic block by flecainide are common to 1795insD, $\Delta \mathrm{KPQ}, \Delta \mathrm{K} 1500$, and E1784K, but not to T1304M. This negative shift of inactivation will reduce the availability of the channels at the resting membrane potential, and increase the proportion of inactivated channels in both the open and closed state, reducing the Na current and increasing the sensitivity to Na-channel blockers. A positive shift in activation is another "loss-of-function" property evident in all the mutants, including T1304M, making it less likely that this specific channel property underlies the mixed clinical phenotypes in LQT3. Other channel properties, such as current decay, recovery from inactivation, slow inactivation or use-dependent block, were not common among 1795insD, $\triangle \mathrm{KPQ}, \Delta \mathrm{K} 1500$, and $\mathrm{E} 1784 \mathrm{~K}$.

A negative shift in inactivation is observed in E1784K, $1795 \mathrm{insD}, \Delta \mathrm{KPQ}$, and $\Delta \mathrm{K} 1500$, and may play a role in the overlap of the LQT3 clinical phenotype with BrS and SND in the mutation carriers, although the number of LQT3 mutations that have been evaluated in this detail is still small, the biophysical and pharmacological properties presented in a cultured cell line may not necessarily reflect the situation in vivo, and the effects of the mutation may be different in ventricular myocytes vs sinus node cells. Further studies that combine clinical and in vitro phenotyping in LQT3 mutations with and without overlapping clinical phenotypes will be required to confirm these findings. Nevertheless, a negative shift in inactivation and enhanced tonic block are common biophysical properties observed among SCN5A mutations in the LQT3/BrS overlapping phenotype.

\section{Conclusions}

E1784K is the most common LQT3 mutation. In patients with this and other LQT3 mutations, overlap of BrS and SND is relatively common. In vitro studies with E1784K and previous reports of LQT3 mutations with and without this clinical overlap syndrome implicate a negative shift in inactivation and enhanced tonic block by drugs as the underlying mechanisms. These findings suggest that patients with LQT3 mutations displaying these characteristics in vitro should not receive class IC drugs. Furthermore, they reinforce the general concept that in vitro characterization of the function of ion channel variants is a key component in generating specific therapeutic strategies for patient management.

\section{Acknowledgments}

The author thanks the following investigators, who were extensively involved in the international project "Functional characterization of the clinical overlap in cardiac sodium channelopathies",1 for collecting clinical and genetic information of the LQT3 families, as well as the functional evaluation of the mutations: Elijah Behr (St George's University of London, UK), Wataru Shimizu (National Cardiovascular Center, Suita, Japan), Shigetomo Fukuhara and Naoki Mochizuki (National Cardiovascular Center Research Institute, Suita, Japan), Minoru Horie (Shiga University of Medical Science, Otsu, Japan), Akihiko Sunami (International University of Health and Welfare, Ohtawara, Japan), Lia Crotti and Peter J. Schwartz (University of Pavia, Pavia, Italy), Eric Schulze-Bahr (University of Muenster, Munster, Germany), and Alfred L. George Jr and Dan M. Roden (Vanderbilt University, Nashville, USA).

\section{References}

1. Catterall WA. From ionic currents to molecular mechanisms: The structure and function of voltage-gated sodium channels. Neuron 2000; 26: 13-25.

2. George AL Jr. Inherited disorders of voltage-gated sodium channels. J Clin Invest 2005; 115: 1990-1999.

3. Bennett PB, Yazawa K, Makita N, George AL Jr. Molecular mechanism for an inherited cardiac arrhythmia. Nature 1995; 376: $683-$ 685.

4. Shimizu W. Clinical impact of genetic studies in lethal inherited cardiac arrhythmias. Circ J 2008; 72: 1926-1936.

5. Makita N, Horie M, Nakamura T, Ai T, Sasaki K, Yokoi H, et al. Drug-induced long-QT syndrome associated with a subclinical SCN5A mutation. Circulation 2002; 106: 1269-1274.

6. Chen Q, Kirsch GE, Zhang D, Brugada R, Brugada J, Brugada P, et al. Genetic basis and molecular mechanism for idiopathic ventricular fibrillation. Nature 1998; 392: 293-296.

7. Schott JJ, Alshinawi C, Kyndt F, Probst V, Hoorntje TM, Hulsbeek $\mathrm{M}$, et al. Cardiac conduction defects associate with mutations in SCN5A. Nat Genet 1999; 23: 20-21.

8. Benson DW, Wang DW, Dyment M, Knilans TK, Fish FA, Strieper $\mathrm{MJ}$, et al. Congenital sick sinus syndrome caused by recessive mutations in the cardiac sodium channel gene (SCN5A). J Clin Invest 2003; 112: 1019-1028.

9. Groenewegen WA, Firouzi M, Bezzina CR, Vliex S, van Langen IM, Sandkuijl L, et al. A cardiac sodium channel mutation cosegregates 
with a rare connexin 40 genotype in familial atrial standstill. Circ Res 2003; 92: 14-22.

10. Makita N, Sasaki K, Groenewegen WA, Yokota T, Yokoshiki H, Murakami T, et al. Congenital atrial standstill associated with coinheritance of a novel SCN5A mutation and connexin 40 polymorphisms. Heart Rhythm 2005; 2: 1128-1134.

11. Takehara N, Makita N, Kawabe J, Sato N, Kawamura Y, Kitabatake A, et al. A cardiac sodium channel mutation identified in Brugada syndrome associated with atrial standstill. J Intern Med 2004; 255: $137-142$.

12. Wang DW, Viswanathan PC, Balser JR, George AL Jr, Benson DW. Clinical, genetic, and biophysical characterization of SCN5A mutations associated with atrioventricular conduction block. Circulation 2002; 105: $341-346$.

13. Wang DW, Desai RR, Crotti L, Arnestad M, Insolia R, Pedrazzini $\mathrm{M}$, et al. Cardiac sodium channel dysfunction in sudden infant death syndrome. Circulation 2007; 115: 368-376.

14. Otagiri T, Kijima K, Osawa M, Ishii K, Makita N, Matoba R, et al. Cardiac ion channel gene mutations in sudden infant death syndrome. Pediatr Res 2008; 64: 482-487.

15. Ackerman MJ, Siu BL, Sturner WQ, Tester DJ, Valdivia CR, Makielski JC, et al. Postmortem molecular analysis of SCN5A defects in sudden infant death syndrome. JAMA 2001; 286: 2264-2269.

16. Olson TM, Michels VV, Ballew JD, Reyna SP, Karst ML, Herron $\mathrm{KJ}$, et al. Sodium channel mutations and susceptibility to heart failure and atrial fibrillation. JAMA 2005; 293: 447-454.

17. Darbar D, Kannankeril PJ, Donahue BS, Kucera G, Stubblefield T, Haines JL, et al. Cardiac sodium channel (SCN5A) variants associated with atrial fibrillation. Circulation 2008; 117: 1927-1935.

18. Ge J, Sun A, Paajanen V, Wang S, Su C, Yang Z, et al. Molecular and clinical characterization of a novel SCN5A mutation associated with atrioventricular block and dilated cardiomyopathy. Circ Arrhythmia Electrophysiol 2008; 1: 83-92.

19. McNair WP, Ku L, Taylor MRG, Fain PR, Dao D, Wolfel E, et al. SCN5A mutation associated with dilated cardiomyopathy, conduction disorder, and arrhythmia. Circulation 2004; 110: 2163-2167.

20. Watanabe H, Koopmann TT, Le Scouarnec S, Yang T, Ingram CR, Schott JJ, et al. Sodium channel beta1 subunit mutations associated with Brugada syndrome and cardiac conduction disease in humans. J Clin Invest 2008; 118: 2260-2268.

21. Keating MT. The long QT syndrome: A review of recent molecular genetic and physiologic discoveries. Medicine (Baltimore) 1996; 75: $1-5$.

22. Wang Q, Shen J, Splawski I, Atkinson D, Li Z, Robinson JL, et al. SCN5A mutations associated with an inherited cardiac arrhythmia, long QT syndrome. Cell 1995; 80: 805-811.

23. Schwartz PJ, Priori SG, Locati EH, Napolitano C, Cantu F, Towbin JA, et al. Long QT syndrome patients with mutations of the SCN5A and HERG genes have differential responses to $\mathrm{Na}^{+}$channel blockade and to increases in heart rate. Implications for gene-specific therapy. Circulation 1995; 92: $3381-3386$.

24. Benhorin J, Taub R, Goldmit M, Kerem B, Kass RS, Windman I, et al. Effects of flecainide in patients with new SCN5A mutation: Mutation-specific therapy for long-QT syndrome? Circulation 2000; 101: $1698-1706$

25. Schwartz PJ. The congenital long QT syndromes from genotype to phenotype: Clinical implications. J Intern Med 2006; 259: 39-47.

26. Brugada P, Brugada J. Right bundle branch block, persistent ST segment elevation and sudden cardiac death: A distinct clinical and electrocardiographic syndrome: A multicenter report. J Am Coll Cardiol 1992; 20: 1391-1396.

27. Wilde AA, Antzelevitch C, Borggrefe M, Brugada J, Brugada R, Brugada P, et al. Proposed diagnostic criteria for the Brugada syndrome. Eur Heart J 2002; 23: 1648 - 1654.

28. Antzelevitch C. The Brugada syndrome: Ionic basis and arrhythmia mechanisms. J Cardiovasc Electrophysiol 2001; 12: 268-272.

29. Tan HL, Bink-Boelkens MT, Bezzina CR, Viswanathan PC, BeaufortKrol GC, van Tintelen PJ, et al. A sodium-channel mutation causes isolated cardiac conduction disease. Nature 2001; 409: 1043-1047.

30. Shirai N, Makita N, Sasaki K, Yokoi H, Sakuma I, Sakurada H, et al. A mutant cardiac sodium channel with multiple biophysical defects associated with overlapping clinical features of Brugada syndrome and cardiac conduction disease. Cardiovasc Res 2002; 53: 348-354.

31. Makiyama T, Akao M, Tsuji K, Doi T, Ohno S, Takenaka K, et al. High risk for bradyarrhythmic complications in patients with Brugada syndrome caused by SCN5A gene mutations. J Am Coll Cardiol 2005; 46: $2100-2106$

32. Veldkamp MW, Viswanathan PC, Bezzina C, Baartscheer A, Wilde AA, Balser JR. Two distinct congenital arrhythmias evoked by a multidysfunctional $\mathrm{Na}^{+}$channel. Circ Res 2000; 86: E91-E97.
33. Bezzina C, Veldkamp MW, van Den Berg MP, Postma AV, Rook MB, Viersma JW, et al. A single $\mathrm{Na}^{+}$channel mutation causing both long-QT and Brugada syndromes. Circ Res 1999; 85: 1206-1213.

34. Viswanathan PC, Bezzina CR, George AL Jr, Roden DM, Wilde AA, Balser JR. Gating-dependent mechanisms for flecainide action in SCN5A-linked arrhythmia syndromes. Circulation 2001; 104: 1200 1205.

35. Priori SG, Napolitano C, Schwartz PJ, Bloise R, Crotti L, Ronchetti E. The elusive link between LQT3 and Brugada syndrome: The role of flecainide challenge. Circulation 2000; 102: 945-947.

36. Moss AJ, Windle JR, Hall WJ, Zareba W, Robinson JL, McNitt S, et al. Safety and efficacy of flecainide in subjects with long QT-3 syndrome ( $\triangle \mathrm{KPQ}$ mutation): A randomized, double-blind, placebo-controlled clinical trial. Ann Noninvasive Electrocardiol 2005; 10: 5966.

37. Grant AO, Carboni MP, Neplioueva V, Starmer CF, Memmi M, Napolitano C, et al. Long QT syndrome, Brugada syndrome, and conduction system disease are linked to a single sodium channel mutation. J Clin Invest 2002; 110: 1201-1209.

38. Veldkamp MW, Wilders R, Baartscheer A, Zegers JG, Bezzina CR, Wilde AAM. Contribution of sodium channel mutations to bradycardia and sinus node dysfunction in LQT3 families. Circ Res 2003; 92: 976-983.

39. Moss AJ, Zareba W, Benhorin J, Locati EH, Hall WJ, Robinson JL, et al. ECG T-wave patterns in genetically distinct forms of the hereditary long QT syndrome. Circulation 1995; 92: 2929-2934.

40. Wei J, Wang DW, Alings M, Fish F, Wathen M, Roden DM, et al. Congenital long-QT syndrome caused by a novel mutation in a conserved acidic domain of the cardiac $\mathrm{Na}^{+}$channel. Circulation 1999; 99: $3165-3171$.

41. Makita N, Behr E, Shimizu W, Horie M, Sunami A, Crotti L, et al. The E1784K mutation in SCN5A is associated with mixed clinical phenotype of type 3 long QT syndrome. J Clin Invest 2008; 118: 2219-2229.

42. Splawski I, Shen J, Timothy KW, Lehmann MH, Priori S, Robinson $\mathrm{JL}$, et al. Spectrum of mutations in long-QT syndrome genes: KVLQT1, HERG, SCN5A, KCNE1, and KCNE2. Circulation 2000; 102: $1178-1185$.

43. Priori SG, Napolitano C, Gasparini M, Pappone C, Della Bella P, Brignole $\mathrm{M}$, et al. Clinical and genetic heterogeneity of right bundle branch block and ST-segment elevation syndrome: A prospective evaluation of 52 families. Circulation 2000; 102: 2509-2515.

44. Shin DJ, Kim E, Park SB, Jang WC, Bae Y, Han J, et al. A novel mutation in the SCN5A gene is associated with Brugada syndrome. Life Sci 2007; 80: 716-724.

45. Juang JM, Huang SK, Tsai CT, Chiang FT, Lin JL, Lai LP, et al. Characteristics of Chinese patients with symptomatic Brugada syndrome in Taiwan. Cardiology 2003; 99: 182-189.

46. Zareba W, Sattari MN, Rosero S, Couderc JP, Moss AJ. Altered atrial, atrioventricular, and ventricular conduction in patients with the long QT syndrome caused by the DKPQ SCN5A sodium channel gene mutation. Am J Cardiol 2001; 88: 1311-1314.

47. Shin DJ, Jang Y, Park HY, Lee JE, Yang K, Kim E, et al. Genetic analysis of the cardiac sodium channel gene SCN5A in Koreans with Brugada syndrome. J Hum Genet 2004; 49: 573-578.

48. Chen T, Inoue M, Sheets MF. Reduced voltage dependence of inactivation in the SCN5A sodium channel mutation delF1617. Am J Physiol Heart Circ Physiol 2005; 288: H2666-H2676.

49. Smits JP, Koopmann TT, Wilders R, Veldkamp MW, Opthof T, Bhuiyan ZA, et al. A mutation in the human cardiac sodium channel $($ E161K) contributes to sick sinus syndrome, conduction disease and Brugada syndrome in two families. J Mol Cell Cardiol 2005; 38: 969-981.

50. Schulze-Bahr E, Eckardt L, Breithardt G, Seidl K, Wichter T, Wolpert C, et al. Sodium channel gene (SCN5A) mutations in 44 index patients with Brugada syndrome: Different incidences in familial and sporadic disease. Hum Mutat 2003; 21: 651-652.

51. Cordeiro JM, Barajas-Martinez H, Hong K, Burashnikov E, Pfeiffer $\mathrm{R}$, Orsino AM, et al. Compound heterozygous mutations P336L and I1660V in the human cardiac sodium channel associated with the Brugada syndrome. Circulation 2006; 114: 2026-2033.

52. Rossenbacker T, Carroll SJ, Liu H, Kuiperi C, de Ravel TJ, Devriendt $\mathrm{K}$, et al. Novel pore mutation in SCN5A manifests as a spectrum of phenotypes ranging from atrial flutter, conduction disease, and Brugada syndrome to sudden cardiac death. Heart Rhythm 2004; 1: $610-615$.

53. Itoh $\mathrm{H}$, Shimizu M, Tanaka S, Mabuchi H, Imoto K. A novel missense mutation in the SCN5A gene associated with Brugada syndrome bidirectionally affecting blocking actions of antiarrhythmic drugs. J Cardiovasc Electrophysiol 2005; 16: 486-493. 
54. Potet F, Mabo P, Le Coq G, Probst V, Schott JJ, Airaud F, et al. Novel brugada SCN5A mutation leading to ST segment elevation in the inferior or the right precordial leads. J Cardiovasc Electrophysiol 2003; 14: 200-203.

55. Smits JP, Eckardt L, Probst V, Bezzina CR, Schott JJ, Remme CA, et al. Genotype-phenotype relationship in Brugada syndrome: Electrocardiographic features differentiate SCN5A-related patients from non-SCN5A-related patients. J Am Coll Cardiol 2002; 40: 350-356.

56. Kyndt F, Probst V, Potet F, Demolombe S, Chevallier JC, Baro I, et al. Novel SCN5A mutation leading either to isolated cardiac conduction defect or Brugada syndrome in a large French family. Circulation 2001; 104: 3081-3086.

57. Tan BH, Valdivia CR, Song C, Makielski JC. Partial expression defect for the SCN5A missense mutation G1406R depends upon splice variant background Q1077 and rescue by mexiletine. Am J Physiol Heart Circ Physiol 2006; 291: H1822-H1828.

58. Kehl HG, Haverkamp W, Rellensmann G, Yelbuz TM, Krasemann T, Vogt J, et al. Life-threatening neonatal arrhythmia: Successful treatment and confirmation of clinically suspected extreme long QTsyndrome-3 [Images in cardiovascular medicine]. Circulation 2004; 109: e205-e206.

59. Chang CC, Acharfi S, Wu MH, Chiang FT, Wang JK, Sung TC, et al. A novel SCN5A mutation manifests as a malignant form of long QT syndrome with perinatal onset of tachycardia/bradycardia. Cardiovasc Res 2004; 64: 268-278.

60. Tester DJ, Will ML, Haglund CM, Ackerman MJ. Compendium of cardiac channel mutations in 541 consecutive unrelated patients referred for long QT syndrome genetic testing. Heart Rhythm 2005; 2: 507-517.

61. Valdivia CR, Ackerman MJ, Tester DJ, Wada T, McCormack J, Ye B, et al. A novel SCN5A arrhythmia mutation, M1766L, with expression defect rescued by mexiletine. Cardiovasc Res 2002; 55: 279-289.

62. Ye B, Valdivia CR, Ackerman MJ, Makielski JC. A common human SCN5A polymorphism modifies expression of an arrhythmia causing mutation. Physiol Genomics 2003; 12: 187-193.

63. Lupoglazoff JM, Cheav T, Baroudi G, Berthet M, Denjoy I, Cauchemez B, et al. Homozygous SCN5A mutation in long-QT syndrome with functional two-to- one atrioventricular block. Circ Res 2001; 89: E16-E21.

64. Tester DJ, Ackerman MJ. Genetic testing for cardiac channelopathies: Ten questions regarding clinical considerations for heart rhythm allied professionals. Heart Rhythm 2005; 2: 675-677.

65. Shim SH, Ito M, Maher T, Milunsky A. Gene sequencing in neonates and infants with the long QT syndrome. Genet Test 2005; 9: 281 284.

66. Baroudi G, Chahine M. Biophysical phenotypes of SCN5A mutations causing long QT and Brugada syndromes. FEBS Lett 2000; 487: $224-228$.

67. Nagatomo T, January CT, Makielski JC. Preferential block of late sodium current in the LQT3 Delta KPQ mutant by the class IC antiarrhythmic flecainide. Mol Pharmacol 2000; 57: 101 - 107. 